\title{
Changes of postural stability according to ankle fixation in healthy subjects
}

\author{
Su-Hyeon Jeong, A-Young Mun, Song-Eun Lee, Min-Ju Kim, Hui-Jin Lee, Kook-Bin Baek, Ki Hun Cho
}

Department of Physical Therapy, Korea National University of Transportation, Jeungpyeong, Republic of Korea

Objective: The purpose of this study was to examine the changes in postural stability according to ankle fixation in healthy university students.

Design: Cross-sectional study.

Methods: Thirty healthy subjects ( 15 males and 15 females, 20.13 years, $167.49 \mathrm{~cm}, 65.87 \mathrm{~kg}$ ) were recruited on a voluntary basis. The BT4 system (HUR Laps Oy, Tampere, Finland) was used to measure the static (standing posture with eyes open and eyes closed) and dynamic (external perturbation and limits of stability (LOS) in the forward, backward, left, and right side) balance abilities. External perturbation was measured by the subject's postural sway velocity and area for 20 seconds after being impacted by a gym ball. Static and dynamic stabilities were measured with ankle joint fixation and non-fixation conditions. Ankle fixation was provided using Mueller tape on both ankle joints.

Results: For static stability under the standing posture, there was no significant difference between standing with ankle joint fixation and non-fixation conditions. However, dynamic stability (external perturbation and LOS in the forward, backward, left, and right side) was significantly higher in the standing with the non-fixation condition compared to the standing with ankle joint fixation condition $(p<0.05)$.

Conclusions: Our results reveal that ankle joint fixation can influence dynamic stability during standing. Thus, we believe that this result provides basic information for making improvements in postural control and may be useful in balance training for fall prevention.

Key Words: Ankle, Falling, Postural balance

\section{Introduction}

Balance is the ability to maintain the center of gravity (COG) within the base of support (BOS) in order to control postural stability [1]. Balance ability is an essential factor for independent activities of daily living, and it decreases with aging. Specifically, lower extremity range of motion, visual sense, and proprioception decreases with aging, which result in increased risk of falls [2]. Balance can be divided into static and dynamic balance. Static balance relates to the ability to stand without any wobbling and maintaining the COG within the BOS, and dynamic balance means the ability to control posture without falling while receiving stimulus from the environment or voluntarily moving [3]. Having postural control is the essential factor in the performance of activities of daily living and functional movements, and it occurs through continuous activation of muscles that are required for maintaining the COG within the BOS [4,5]. Postural strategies to maintain balance and the ability to activate sufficient muscle strength during movements are important factors in postural control [6].

The human body uses ankle, hip, and step strategies to maintain balance [5], and balance control in static postures occur through ankle and hip strategies [7]. Ankle strategy is

Received: 26 February, 2019 Revised: 12 March, 2019 Accepted: 14 March, 2019

Corresponding author: Ki Hun Cho (ORCID https://orcid.org/0000-0001-6248-1768)

Department of Physical Therapy, Korea National University of Transportation, 61 Deahak-ro, Jeungpyeong-gun, Chungbuk 27909, Republic of Korea Tel: 82-43-820-5206 Fax: 82-43-820-5202 E-mail: mamiya34@gmail.com

(c) This is an Open-Access article distributed under the terms of the Creative Commons Attribution Non-Commercial License (http://creativecommons.org/licenses/ by-nc/4.0) which permits unrestricted non-commercial use, distribution, and reproduction in any medium, provided the original work is properly cited.

Copyright (๑ 2019 Korean Academy of Physical Therapy Rehabilitation Science 
recovering standing balance through contraction of the muscles around the ankle joint [8], and it is the first control strategy during postural control. It is usually used on firm surfaces with few disturbances [9]. When ankle strategy is inhibited, the ankle muscles have difficulties in producing sufficient contraction. It is reported that this inhibition decreases postural control, and induces an increase in disturbance and a decrease in balance ability, resulting in increased risk of falls [6].

According to previous studies that analyzed the effects of fixation of the ankle and knee joints on static balance, the fixation of ankle and knee joints induced a decrease in postural control in standing position [10]. However, other studies showed that ankle joint fixation leads to increased postural stability in standing position [11]. Previous studies have used various methods to fixate the ankle joint, making it difficult to generalize the results. In addition, since previous studies $[10,11]$, have focused only on the changes in static stability during standing with ankle fixation, studies on the effect of ankle fixation on dynamic stability are needed. Therefore, the purpose of this study is to examine the changes in static and dynamic postural stability according to ankle fixation in healthy university students.

\section{Methods}

\section{Subjects}

A total of thirty young male and female subjects volun-

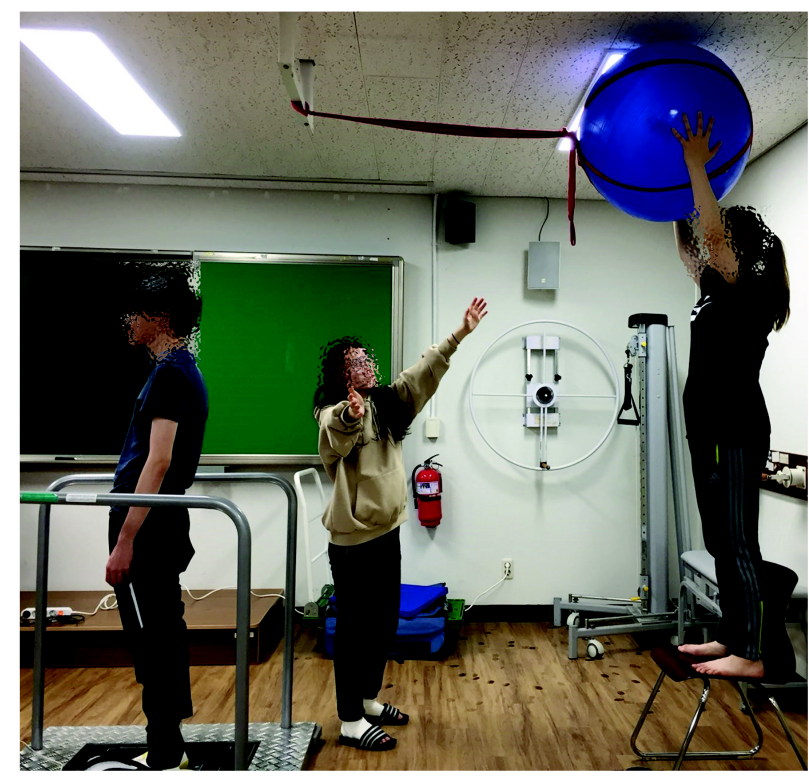

Figure 1. Setting for the measurement of external perturbation. tarily agreed to participate in the study (15 males, 20.13 years, $167.49 \mathrm{~cm}, 65.87 \mathrm{~kg})$. The inclusion criteria were as follows: (1) those with no balance or vestibular disorders and (2) no weakness or pain in the lower extremity. The exclusion criteria was having experience with leg surgery. The subjects agreed to participate in the study and provided their informed consent after being fulling explained of the procedure. The study was approved by the Institutional Review Board of the Korea University of transportation (IRB No. KNUT IRB 2018-08). The sample size was determined after a calculation based on results of the G-power 3.1.9.3 software (G Power Team, Dusseldorf, Germany). The power and alpha were set at 0.95 and 0.05 , respectively, and the effect size was set at 0.63 according to a priori analysis, with at least 29 subjects required. Thus, 30 participants were enrolled with the consideration for potential dropouts.

\section{Procedures}

This study was a cross-sectional single-group repeatedmeasures design. For measurements of static stability, external perturbation, and for dynamic stability, a computerized balance platform (BT4; HUR Labs Oy, Tampere, Finland) was used [12]. Postural sway velocity and area was assessed with the eyes open and eyes closed condition while standing on the balance platform for 30 seconds for static stability [13]. The subject stood on the balance platform with their legs shoulder-width apart and looked straight ahead. In order to measure postural sway, the subject was asked to stand quietly in a comfortable upright position on the force

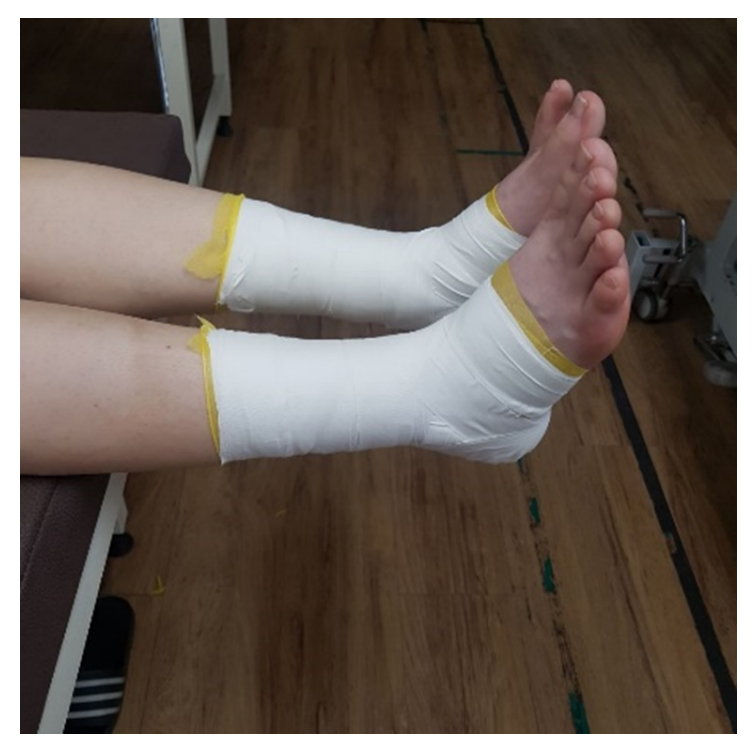

Figure 2. Ankle fixation using muller tape. 
plate while looking straight ahead. Three repetitions of each measurement were performed and the average was used for statistical analysis. A resting time of 3 minutes was provided between measurements. In addition, the external perturbation was provided by a gym ball hanging from the ceiling. For external perturbation, a gym ball fell freely from the ceiling and made impact onto the subject's body while the subject stood on a balance platform (Figure 1). The position where the ball began to fall and the position where the ball contacted the subject's low back were equal for all subjects. The external perturbation effect was measured by the subject's postural sway velocity and area for 20 seconds after being impacted by the gym ball. As for the external perturbation, 10 seconds (before and after 5 seconds at the time external perturbation occurred by the gym ball) were excluded from the 30-second measurement time frame. Dynamic stability was measured with limits of stability (LOS) in the forward, backward, left, and right side for 8 seconds, re- spectively [13]. When the LOS were measured, the subject's feet were aligned along the marked point of the medial malleolus printed on the platform, and their center of pressure was placed as a curve shown in the first position on a computer monitor. They were asked to move their weight along each direction (one direction at a time) according to the appearance of a target on the monitor as soon as possible (using the ankle joint according to the primary axis of movement, without changing the foot position) [14]. All measurements were performed twice with the ankle fixation and the ankle non-fixation conditions. Ankle fixation was provided using Mueller tape (Mueller sports medicine, Madison, WI, USA) in both ankle joints and taping was performed based on the published guidelines (Figure 2) [15].

\section{Data analysis}

The data collected through this study were compared with the mean and standard deviation of each variable using

Table 1. General characteristic of the subjects

$(\mathrm{N}=30)$

\begin{tabular}{|c|c|c|c|}
\hline Parameter & Male $(n=15)$ & Female $(n=15)$ & Total $(n=30)$ \\
\hline Age (y) & $20.06(0.25)$ & $20.20(0.56)$ & $20.13(0.43)$ \\
\hline Height $(\mathrm{cm})$ & $172.23(5.62)$ & $162.76(5.03)$ & $167.49(7.11)$ \\
\hline Weight (kg) & $72.65(9.22)$ & $59.09(9.91)$ & $65.87(11.66)$ \\
\hline Body mass index $\left(\mathrm{kg} / \mathrm{m}^{2}\right)$ & $21.18(7.35)$ & $32.36(7.54)$ & $26.77(9.27)$ \\
\hline
\end{tabular}

Values are presented as mean (SD).

Table 2. Changes in postural stability according to ankle fixation and non-fixation conditions

$(\mathrm{N}=30)$

\begin{tabular}{|c|c|c|c|c|}
\hline Parameter & Ankle non-fixation & Ankle fixation & Changes & $\mathrm{t}(p)$ \\
\hline \multicolumn{5}{|l|}{ Static stability } \\
\hline $\operatorname{EOA}\left(\mathrm{mm}^{2}\right)$ & $287.43(209.42)$ & $255.64(189.96)$ & $31.79(146.28)$ & $1.190(0.244)$ \\
\hline $\operatorname{ECA}\left(\mathrm{mm}^{2}\right)$ & $292.95(203.24)$ & $277.51(193.76)$ & $15.43(188.26)$ & $0.449(0.657)$ \\
\hline $\mathrm{EOV}(\mathrm{mm} / \mathrm{s})$ & $8.81(3.14)$ & $8.35(2.73)$ & $0.46(1.94)$ & $1.302(0.203)$ \\
\hline $\operatorname{ECV}(\mathrm{mm} / \mathrm{s})$ & $9.94(3.05)$ & $9.53(2.61)$ & $0.40(2.18)$ & $1.023(0.315)$ \\
\hline \multicolumn{5}{|c|}{ External perturbation } \\
\hline $\operatorname{PVEA}\left(\mathrm{mm}^{2}\right)$ & $1,052.92(666.38)$ & $823.39(415.21)$ & $229.52(630.65)$ & $1.993(0.056)$ \\
\hline $\operatorname{PVEP}(\mathrm{mm} / \mathrm{s})$ & $19.97(4.12)$ & $16.33(3.77)$ & $3.64(4.85)$ & $4.111(<0.001)$ \\
\hline \multicolumn{5}{|l|}{ Dynamic stability } \\
\hline LOS-F $\left(^{\circ}\right)$ & $5.42(1.70)$ & $4.70(1.83)$ & $0.71(0.96)$ & $4.069(<0.001)$ \\
\hline LOS-B $\left(^{\circ}\right)$ & $3.79(1.45)$ & $3.44(1.55)$ & $0.34(0.76)$ & $2.470(0.020)$ \\
\hline LOS-L $\left(^{\circ}\right)$ & $4.23(1.09)$ & $3.86(1.13)$ & $0.36(0.96)$ & $2.082(0.046)$ \\
\hline LOS-R $\left(^{\circ}\right)$ & $4.62(0.97)$ & $4.22(0.97)$ & $0.39(0.79)$ & $2.718(0.011)$ \\
\hline
\end{tabular}

Values are presented as mean (SD).

EOA: eye open postural sway area, ECA: eye close postural sway area, EOV: eye open postural sway velocity, ECV: eye close postural sway velocity, PVEA: postural sway area on external perturbation under the standing posture, PVEP: postural sway velocity on external perturbation under the standing posture, LOS-F: limit of stability on front, LOS-B: limit of stability on backward, LOS-L: limit of stability on left side, LOS-R: limit of stability on right side. $p<0.05$. 


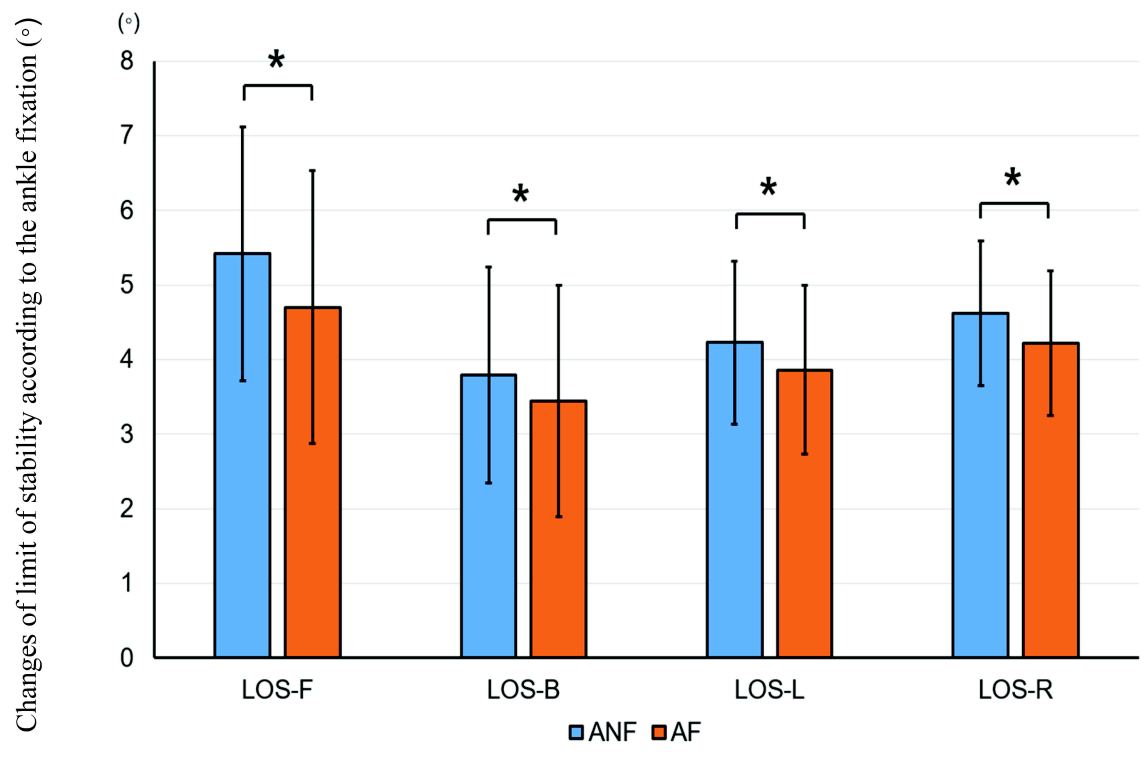

Figure 3. Changes of limit of stability according to the ankle fixation. LOS: limit of stability, F: forward, B: backward, L: left side, R: right side, ANF: ankle non fixation, AF: ankle fixation. ${ }^{*} p<0.05$.
PASWStatistics ver. 22.0 (IBM Co., Armonk, NY, USA). For dependent variable measures, the paired t-test was used to compare dependent variables within groups. A significance level of 0.05 was used for all tests.

\section{Results}

A summary of the general characteristics at enrollment is listed in Table 1. Changes in static and dynamic postural stabilities according to the ankle fixation are shown in Table 2 .

For static stability, there was no significant difference between the standing with ankle joint fixation and non-fixation conditions. However, with external perturbation provided during standing, dynamic stability was significantly higher in the standing with ankle joint non-fixation condition than the standing with ankle joint fixation condition $(p<0.05)$.

\section{Discussion}

Postural control is placing the COG on top of the BOS, and stable balance may be maintained when the COG is perpendicular to the standing surface. However, proper modification of posture is required when the COG is near the limitations of stability [16]. Therefore, this study measured the changes in static and dynamic postural stability with fixation of the ankle joint using tape. As a result, there was no significant difference between the standing with ankle joint fixation and non-fixation conditions for static stability. However, with external perturbation provided under the standing posture, dynamic stability was significantly higher in the standing with ankle joint non-fixation condition than the fixation condition $(p<0.05)$.

Healthy adults use the ankle, hip, and step strategies to maintain stability in various directions to respond to external stimuli. When disturbances of balance are relatively small and the supporting surface is stabilized, movements produced from the ankle strategy are used to maintain postural stability [17]. According to a previous study, the LOS is shaped in a conical form, where the maximal anterior angle is 6-8 degrees and the maximal external angle is 8 degrees [18]. In the result of this study, near normal range angles was observed in the ankle non-fixation condition $(5.42,3.79$, 4.23, and 4.62 degrees for forward, backward, left, and right side of LOS respectively). However, in the ankle fixation condition, LOS has decreased to $4.70,3.44,3.86$, and 4.22 degrees for forward, backward, left, and right side of the LOS respectively (Figure 3). In contrast, in the measurement of static stability and external perturbation, there was a greater decrease in postural stability in the ankle fixation condition compared to the non-fixation condition. The results of this study may suggest that ankle fixation is effective for enhancing postural stability while maintaining posture against external disturbances and static position. In addition, in a dynamic state requiring more ankle joint movement, ankle fixation may restrict the LOS. Therefore, the fact that taping for ankle fixation could decrease the LOS and increase the risk of falls must be considered in clinical settings.

This study had some limitations. Not only is postural 
strategy needed for maintaining postural stability in standing position, but also appropriate muscle contractions, control of the nervous system, vision and proprioception are required [19]. This study did not control these factors and therefore, future studies need to consider these various factors that may affect postural stability. In addition, this study was conducted on healthy adults. Thus, in the future, patient-based studies are needed.

\section{Acknowledgements}

This was supported by Korea National University of Transportation in 2019 .

\section{Conflict of Interest}

The authors declared no potential conflicts of interest with respect to the authorship and/or publication of this article.

\section{References}

1. Umphred DA, Lazaro RT, Roller M, Burton G. Neurological rehabilitation. 6th ed. London: Elsevier Health Sciences; 2013.

2. Siqueira CM, Lahoz Moya GB, Caffaro RR, Fu C, Kohn AF, Amorim CF, et al. Misalignment of the knees: does it affect human stance stability. J Bodyw Mov Ther 2011;15:235-41.

3. Fransson PA, Kristinsdottir EK, Hafström A, Magnusson M, Johansson R. Balance control and adaptation during vibratory perturbations in middle-aged and elderly humans. Eur J Appl Physiol 2004;91:595-603.

4. Boyas S, Remaud A, Bisson EJ, Cadieux S, Morel B, Bilodeau $\mathrm{M}$. Impairment in postural control is greater when ankle plantarflexors and dorsiflexors are fatigued simultaneously than when fatigued separately. Gait Posture 2011;34:254-9.

5. Johansson R, Magnusson M. Human postural dynamics. Crit
Rev Biomed Eng 1991;18:413-37.

6. Yaggie JA, McGregor SJ. Effects of isokinetic ankle fatigue on the maintenance of balance and postural limits. Arch Phys Med Rehabil 2002;83:224-8.

7. Mackey DC, Robinovitch SN. Mechanisms underlying age-related differences in ability to recover balance with the ankle strategy. Gait Posture 2006;23:59-68.

8. Horak FB, Nashner LM. Central programming of postural movements: adaptation to altered support-surface configurations. J Neurophysiol 1986;55:1369-81.

9. Shumway-Cook A, Woollacott M. Attentional demands and postural control: the effect of sensory context. J Gerontol A Biol Sci Med Sci 2000;55:M10-6.

10. Hwang SJ, Woo YK, Jeon HS. Effects of immobilization of the ankle and knee joints on postural stability in standing. Phys Ther Korea 2008;15:30-7.

11. Kim YG, Lee GH, Lee CS. The effect of wearing an ankle support on the activity of ankle muscles and standing posture balance for stroke patients. Exerc Sci 2013;22:11-21.

12. Gim MN, Lee SB, Yoo KT, Bae JY, Kim MK, Choi JH. The effect of olfactory stimuli on the balance ability of stroke patients. J Phys Ther Sci 2015;27:109-13.

13. Kim MK, Yoo KT. The effects of open and closed kinetic chain exercises on the static and dynamic balance of the ankle joints in young healthy women. J Phys Ther Sci 2017;29:845-50.

14. Kim MK, Kong BS, Yoo KT. The effect of shoe type on static and dynamic balance during treadmill walking in young healthy women. J Phys Ther Sci 2017;29:1653-7.

15. Abell BA. Taping and wrapping made simple. Philadelphia: Wolters Kluwer Health/Lippincott Williams \& Wilkins; 2010.

16. Horak FB. Postural orientation and equilibrium: what do we need to know about neural control of balance to prevent falls? Age Ageing 2006;35 Suppl 2:ii7-11.

17. Jacobs JV, Horak FB. External postural perturbations induce multiple anticipatory postural adjustments when subjects cannot pre-select their stepping foot. Exp Brain Res 2007;179:29-42.

18. Nichols DS. Balance retraining after stroke using force platform biofeedback. Phys Ther 1997;77:553-8.

19. Blenkinsop GM, Pain MTG, Hiley MJ. Balance control strategies during perturbed and unperturbed balance in standing and handstand. R Soc Open Sci 2017;4:161018. 